\title{
nature neurosciencegateway
}

\section{FEATURED ARTICLES}

\section{Death and axons}

Neuroscience Gateway (June 2007) | doi:10.1038/aba1749

High-throughput proteomics identifies a lectin important in the degeneration of neuronal processes.

Like retractable cords returning to the base of a hair dryer, neuronal processes degenerate as a result of injury or neurodegenerative disease. If neuronal cell death follows a molecular program, what is the mechanism of axon degeneration? Plachta et al. report that the carbohydratebinding protein galectin-1 induces neuronal process degeneration in a recent article in Nature

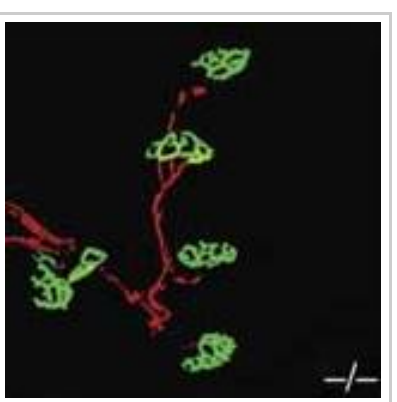
Neuroscience.

The nerve growth factor receptor p75NTR inhibits the extension of neuron processes. Its expression is elevated in embryonic stem cells during neuronal differentiation but declines before process extension. Overexpression of p75NTR causes neuronal death.

In embryonic stem (ES) cells, the authors targeted p75NTR expression to the Mapt locus (encoding tau), which is active during neuronal differentiation and process extension. ES cells expressing the Mapt:p75 $5^{N T R}$ transgene showed normal process extension two days after plating. However, on the fourth day, when neurites in control cultures showed increased branching, neurites in the Mapt:p75 $5^{\text {NTR }}$ cells were fragmented. Degeneration began in secondary processes before affecting primary processes and culminated in neuronal death on day five. Caspases are important in programmed cell death. Although caspase inhibitors blocked neuronal death, they did not block the degeneration of neural processes in cells expressing the Mapt:p75 ${ }^{N T R}$ transgene.

To identify regulators of neurite degeneration, the authors did a high-throughput proteomic analysis of ES cells expressing the Mapt:p75 $75^{N R}$ transgene two days after plating, before any signs of degeneration. They found increased expression of the galactoside-binding protein galectin-1 in transgene expressing cells.

Galectin-1 induces neuronal process degeneration in vitro. Twenty-four hours after galectin-1 treatment, most processes degenerated in differentiated ES cells and primary cultures of cortical neurons. Similar to cells expressing the Mapt:p75 ${ }^{N T R}$ transgene, galectin-1 induced degeneration in secondary processes before primary processes and culminated in neuron death.

p75NTR promotes galectin-1 expression through c-Jun $\mathrm{N}$-terminal kinase (JNK) activity. Expression of the gene encoding galectin-1, Lgals 1, paralleled the expression of p75NTR, which signals through JNK. Phosphorylation of JNK also paralleled the expression of p75NTR. The JNK inhibitor CEP-1347 blocked Mapt: $p 75^{N T R}$ - but not galectin-1-induced degeneration of neuronal 
Galectin-1 also induces degeneration in vivo. The NMDA receptor agonist ibotenic acid kills neurons through a p75NTR-mediated mechanism. Septal injections of ibotenic acid increased galectin-1 expression in p75NTR-positive neurons. However, co-injection of either an antibody specific to galectin-1 or CEP-1347 reduced ibotenic acid-induced cell loss, suggesting that galectin-1 mediates this neurodegeneration.

Axotomy increases p75NTR expression in nerve endings. The authors found increased galectin-1 in axotomized nerve terminals. Motor neuron degeneration was delayed but not prevented in Lgals1 knockout relative to wild-type mice, suggesting that galectin-1 is involved in but not solely responsible for nerve degeneration in vivo.

Spinal cord motor neurons show galectin-1-positive inclusions in people with amyotrophic lateral sclerosis (ALS). Perhaps galectin1 antibodies would delay neurodegeneration in people with ALS and other motor neuron diseases.

Debra Speert,

1. N.Plachtaet al.Identification of a lectin causing the degeneration of neuronal processes using engineered embryonic stem cells.Nature Neuroscience.10,712719(2007) | Article | 